\title{
Realization of Ultraflat Plastic Film Using Dressed-Photon-Phonon-Assisted Selective Etching of Nanoscale Structures
}

\author{
Takashi Yatsui, Wataru Nomura, and Motoichi Ohtsu \\ School of Engineering, The University of Tokyo, 2-11-16 Yayoi, Bunkyo-ku, Tokyo 113-8656, Japan \\ Correspondence should be addressed to Takashi Yatsui; yatsui@ee.t.u-tokyo.ac.jp
}

Received 30 September 2014; Accepted 6 November 2014

Academic Editor: Chennupati Jagadish

Copyright (C) 2015 Takashi Yatsui et al. This is an open access article distributed under the Creative Commons Attribution License, which permits unrestricted use, distribution, and reproduction in any medium, provided the original work is properly cited.

We compared dressed-photon-phonon (DPP) etching to conventional photochemical etching and, using a numerical analysis of topographic images of the resultant etched polymethyl methacrylate (PMMA) substrate, we determined that the DPP etching resulted in the selective etching of smaller scale structures in comparison with the conventional photochemical etching. We investigated the wavelength dependence of the PMMA substrate etching using an $\mathrm{O}_{2}$ gas. As the dissociation energy of $\mathrm{O}_{2}$ is $5.12 \mathrm{eV}$, we applied a continuous-wave $(\mathrm{CW}) \mathrm{He}-\mathrm{Cd}$ laser $(\lambda=325 \mathrm{~nm}, 3.81 \mathrm{eV})$ for the DPP etching and a 5th-harmonic Nd:YAG laser $(\lambda=213 \mathrm{~nm}, 5.82 \mathrm{eV})$ for the conventional photochemical etching. From the obtained atomic force microscope images, we confirmed a reduction in surface roughness, $R_{a}$, in both cases. However, based on calculations involving the standard deviation of the height difference function, we confirmed that the conventional photochemical etching method etched the larger scale structures only, while the DPP etching process selectively etched the smaller scale features.

\section{Introduction}

Organic materials are flexible in comparison with inorganic substrates; therefore, they are important materials as regards the development of future wearable devices [1]. Recently, organic substrates with microstructures have been prepared by mechanical pressing, an injection molding method [2], and the surface roughness values of the obtained materials have been determined by use of a pressing mold. However, because the resultant plastic is soft, these organic substrates cannot be flattened using a mechanical polishing method. Furthermore, although plastic substrates are easy to fabricate in three-dimensional structures, the side walls of these structures cannot be flattened.

In order to achieve an ultraflat surface on an inorganic material, many groups have developed a chemical mechanical polishing (CMP) method [3], resulting in a subnanometer scale flattened surface [4]. However, since CMP is a contact method, it leads to the formation of scratches or digs during polishing, while chemical materials in the slurry penetrate the polished surface, resulting in performance degradation.
Therefore, we have developed a dressed-photon-phonon (DPP) etching technique [5] as a noncontact flattening method. In this process, the free photons of propagating light are coupled with material excitation, including electrons and holes, of the nanoscale material structures; in other words, the photons interacting with the nanoscale material dress the material excitation to form a dressed photon (DP) quasiparticle. The DP also couples with multiple-mode coherent phonons in the material, which act as quasiparticle of DP and phonons (DPP). Therefore, the DPP energy is higher than that of the incident free photon; that is, the DPP realizes energy upconversion [6]. By using the energy upconversion mechanism, selective etching of the nanoscale structure can be successfully realized. Previously, we obtained ultraflat surfaces on substrates including glass [5], diamond, and GaN [7]. Since DPP etching does not require a contact pad, three-dimensional structures have also been flattened [8].

Here, we compare the effectiveness of the DPP etching technique to that of conventional photochemical etching. To evaluate the effectiveness of the DPP etching, we perform 


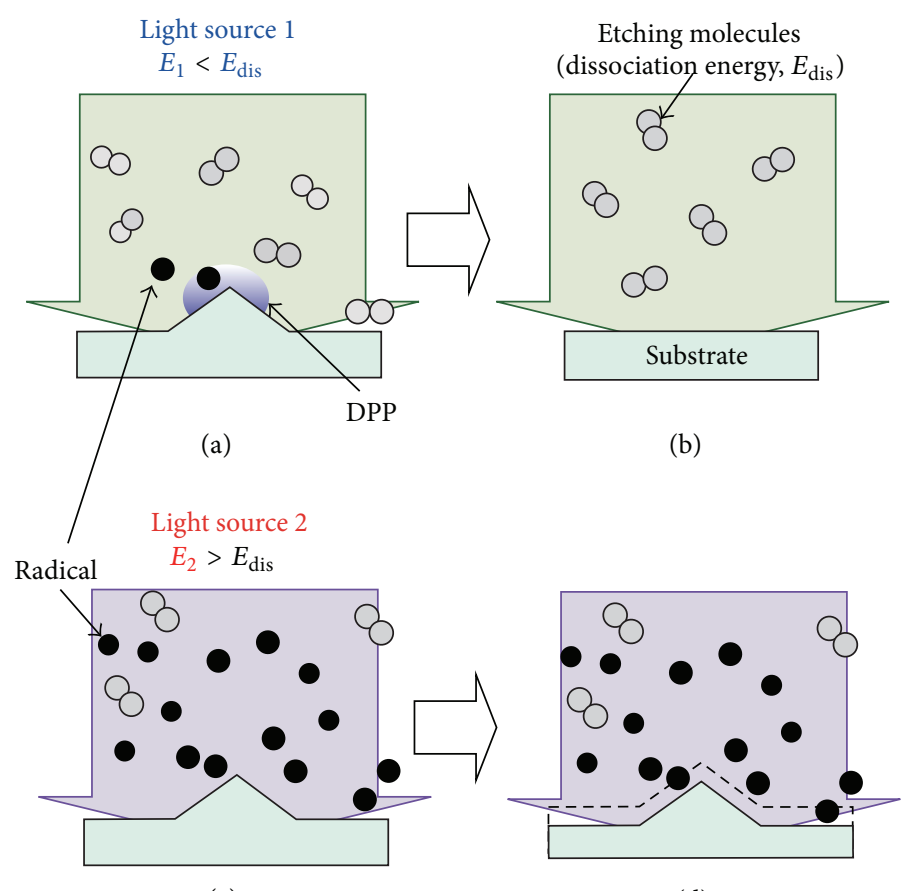

(c)

(d)

Figure 1: ((a) and (b)) DPP etching and ((c) and (d)) conventional photochemical etching process diagrams.

calculations based on the standard deviation of the height difference function in addition to the surface roughness, $R_{a}$. Here, we observe the wavelength dependence of photochemical etching on polymethyl methacrylate (PMMA) substrate as a plastic material.

\section{DPP Etching}

To realize DPP etching, the photon energy of the light source, $E_{1}$, must be lower than the dissociation energy of the etching molecule, $E_{\text {dis }}$. Therefore, the incident photon cannot dissociate the etching molecules in a gas phase. However, the DPP is generated at the apex of the protrusions of the surface. Furthermore, since the DPP energy, $E_{\mathrm{DPP}}$, is larger than that of $E_{1}$, the etching molecules are dissociated selectively when they enter the proximity of the protrusions (Figure 1(a)). Consequently, the dissociated radical molecules etch away at the protrusions and, thus, the DPP etching process automatically stops when the surface is sufficiently flattened (Figure 1(b)).

In contrast to DPP etching, when conventional plasma etching or conventional photochemical etching is used, the photon energy of the light source, $E_{2}$, is higher than $E_{\text {dis }}$, and the etching molecules are dissociated in all directions (Figure 1(c)). Therefore, the dissociated radical atoms etch the substrate with no position dependence (Figure 1(d)).

\section{Experiment}

In this study, we used a PMMA substrate prepared by injection molding in an oxygen atmosphere. Because the dissociation energy of $\mathrm{O}_{2}$ is $5.12 \mathrm{eV}$ [9], we used a continuous-wave (CW) He-Cd laser $(\lambda=325 \mathrm{~nm} ; 3.81 \mathrm{eV}$; excitation power: $0.8 \mathrm{~W} / \mathrm{cm}^{2}$ ) for DPP etching and a 5th-harmonic YAG laser $(\lambda=213 \mathrm{~nm} ; 5.82 \mathrm{eV} ; 20 \mathrm{~Hz}$; pulse width: $5 \mathrm{~ns})$ for conventional photochemical etching. The surface structure was evaluated using an atomic force microscope (AFM) with a "sampling intelligent scan" mode (Hitachi-Hitech-Science Corp.). The scanned area was $1 \times 1 \mu \mathrm{m}$ and incorporated $256 \times$ 256 pixels (a spatial resolution of $4 \mathrm{~nm}$ ) and the AFM images were obtained using tilt compensation and the third-order least-squares method.

\section{Results and Discussion}

Figures 2(a) and 2(b) are AFM images of the surface before and after DPP etching, respectively. It was found that the surface roughness, $R_{a}$, was reduced from 0.30 to $0.22 \mathrm{~nm}$ following 120 min DPP etching (Figure 3). Similar $R_{a}$ reduction was observed following conventional photochemical etching, as can be seen from the AFM images taken before (Figure 2(c), $R_{a}=0.26 \mathrm{~nm}$ ) and after (Figure 2(d), $R_{a}=$ $0.20 \mathrm{~nm}$ ) etching, and comparison between both methods is given in Figure 3. It can be seen that the usage of conventional photochemical etching resulted in a dramatic decrease in etching time.

To investigate the surface morphology in detail, we used the method developed in our previous reports [7]. Instead of examining the value of $R_{a}$ only, which is the average value of the absolute surface height deviations from the best-fitting plane (Figure 4(a)), we used the standard deviation of the height difference function. Here, the $R_{a}$ value 


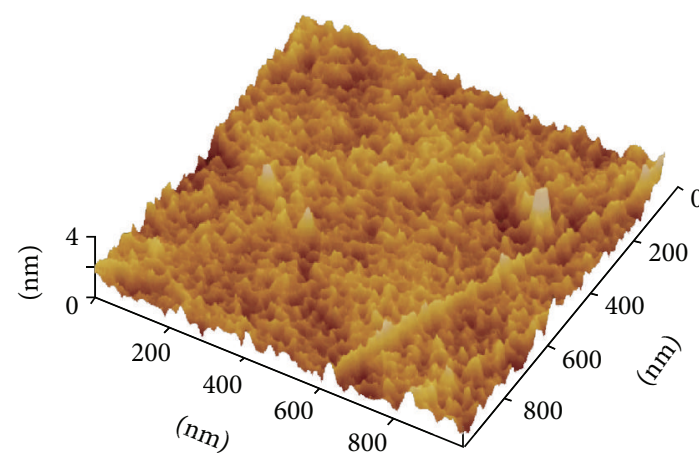

(a)

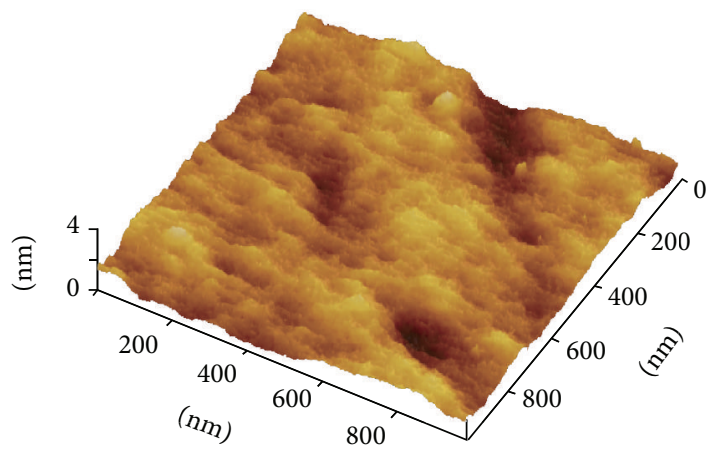

(c)

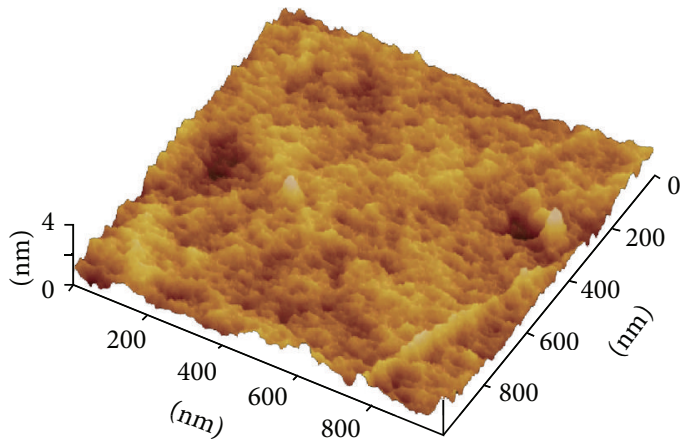

(b)

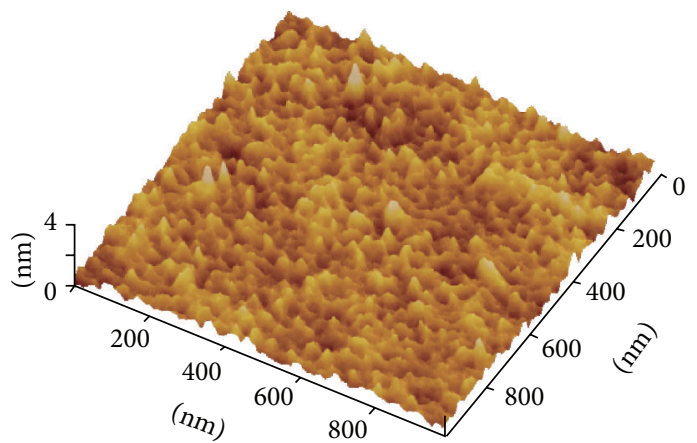

(d)

Figure 2: Typical AFM images of PMMA substrate (a) before and (b) after DPP etching using He-Cd laser $(\lambda=325 \mathrm{~nm}, 3.81 \mathrm{eV})$. Typical AFM images of PMMA substrate (c) before and (d) after conventional photochemical etching using 5th-harmonic YAG laser $(\lambda=213 \mathrm{~nm}$, $5.82 \mathrm{eV})$.

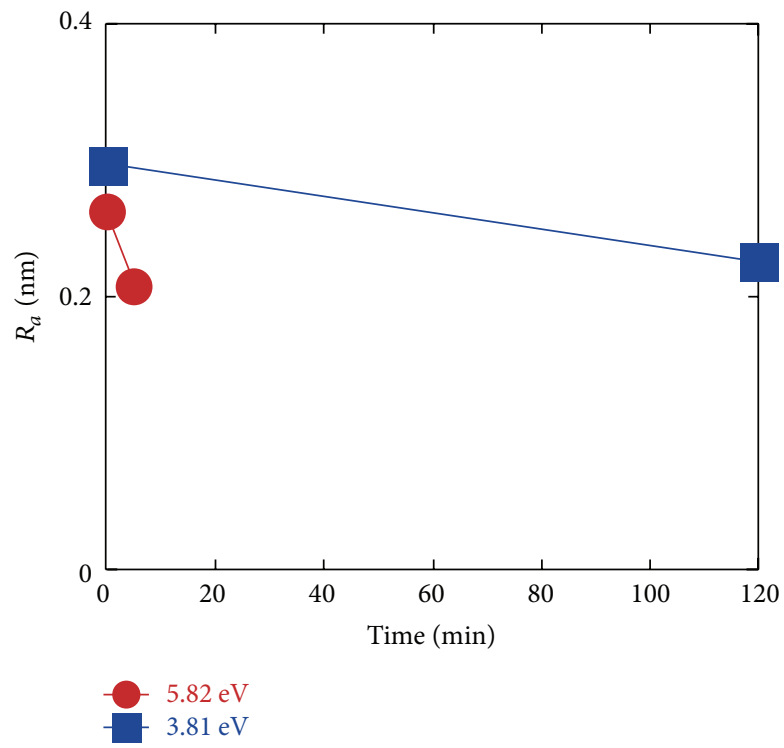

Figure 3: Etching time dependence of surface roughness, $R_{a}$. Blue solid squares correspond to $R_{a}$ using $3.81 \mathrm{eV} \mathrm{He}-\mathrm{Cd}$ laser (DPP etching). Red solid circles correspond to $R_{a}$ using $5.82 \mathrm{eV}$ 5th-harmonic Nd:YAG laser (conventional photochemical etching).

provides information about the average surface roughness for the entire scanning region. However, the standard deviation of the height difference function is given by $R(l)=$ $\sqrt{\left\langle\left(\overline{z_{k+1}^{(l)}}-\overline{z_{k}^{(l)}}\right)^{2} / 2\right\rangle}$, where $l$ is the scale size, $z$ is the height from the best-fitting plane, and $\overline{z_{k}^{(l)}}$ is the average $z$ value of the scale (Figure 4(b)) [10]. This $R(l)$ can be used to determine the contributions of the surface roughness values (at different length scales) to the overall surface roughness. As this value 


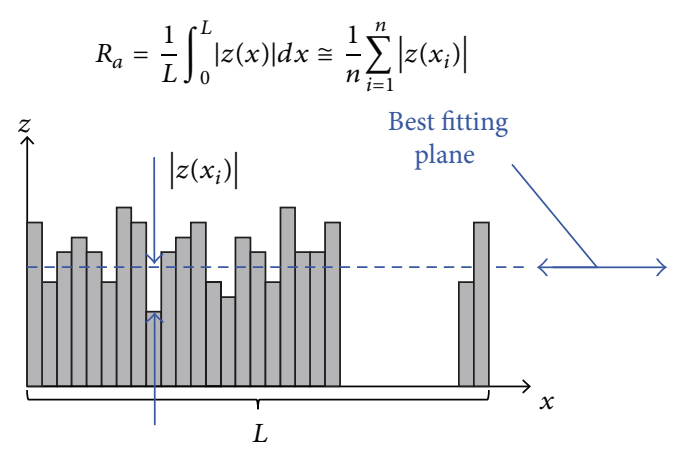

(a)

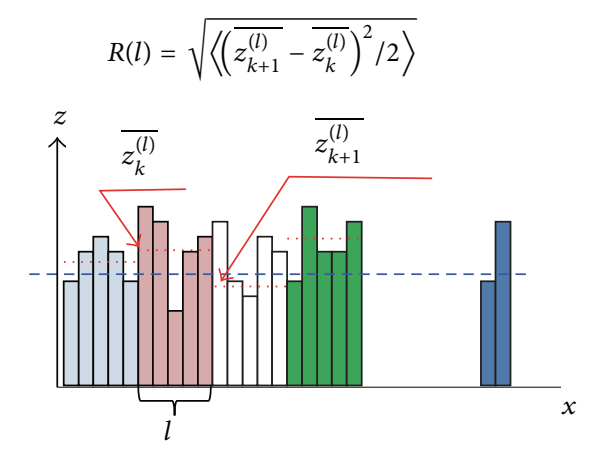

(b)

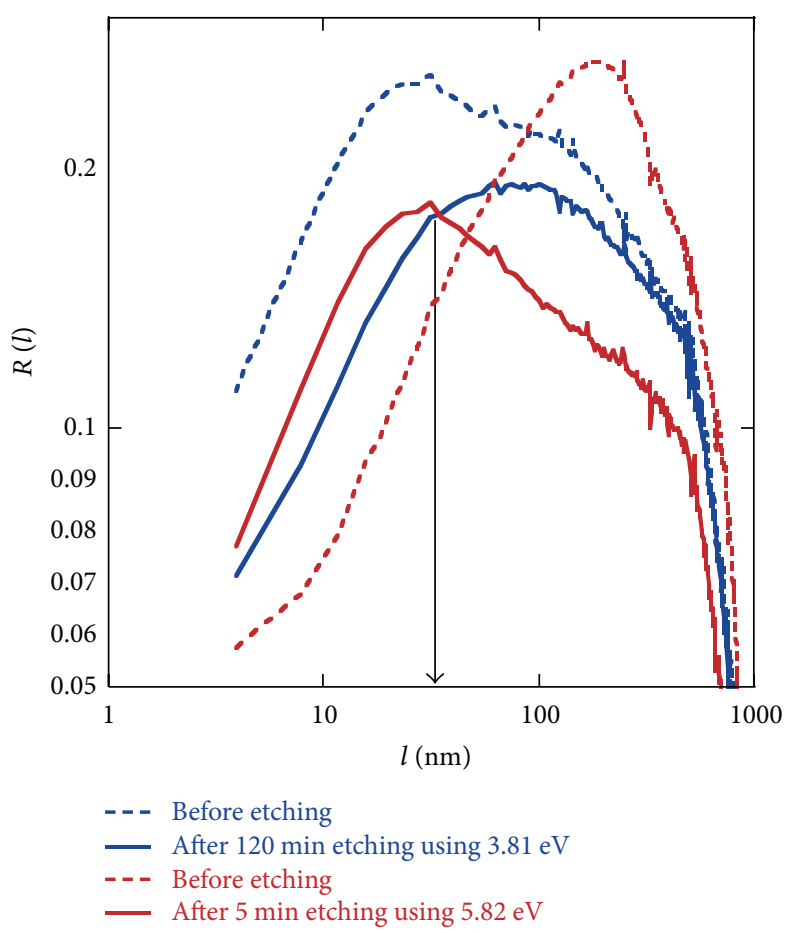

(c)

Figure 4: Schematic of (a) the surface roughness, $R_{a}$, and the standard deviation of the height difference function, $R(l)$. (c) Etching time dependence of the standard deviation of $R(l)$. The blue dashed curve corresponds to Figure 2(a) (before etching); the blue solid curve corresponds to Figure 2(b) (after 120 min etching using 3.81 eV DPP etching); the red dashed curve corresponds to Figure 2(c) (before etching); the red solid curve corresponds to Figure 2(d) (after 5 min etching using $5.82 \mathrm{eV}$ conventional photochemical etching).

indicates the height difference of the next scale, it is known as a two-sample variance. This value is known as the Allan variance, and it is a measure of frequency stability in clocks, oscillators, and amplifiers.

Figure 4 shows the calculated $R(l)$ values from the AFM images. The maximum values of the blue curves decreased from 0.25 to $0.19 \mathrm{~nm}$, which are comparable to the $R_{a}$ values of Figure 2(a) $(0.30 \mathrm{~nm})$ and Figure 2(b) $(0.22 \mathrm{~nm})$ and indicate the results of the DPP etching. Similarly, the maximum values of the red curves decreased from 0.27 to $0.18 \mathrm{~nm}$, which are comparable to the $R_{a}$ values of Figure $2(\mathrm{c})(0.26 \mathrm{~nm})$ and Figure 2(d) $(0.20 \mathrm{~nm})$ and are the photochemical etching results. It is notable that even though the $R_{a}$ values vary significantly with each other, we can therefore confirm that the conventional etching etched on a scale, $l$, larger than
$100 \mathrm{~nm}$, while the DPP etching etched on a smaller scale, with $l$ less than $100 \mathrm{~nm}$. These results therefore establish that DPP etching selectively etches on a smaller scale, as the $R(l)$ value for the DPP etched is smaller than that of the conventional photochemical etching at $l<35 \mathrm{~nm}$ (indicated by the arrow in Figure 4). The increase in $R(l)$ at the smaller scale for the conventional photochemical etching case might be caused by ablation due to the higher photon energy used in this method, in comparison with that of the chemical bonding of PMMA [11].

\section{Conclusion}

Through an experiment on the wavelength-dependent etching of a polymethyl methacrylate (PMMA) substrate and 
calculations based on the standard deviation of the height difference function of the resultant surface, we determined that dressed-photon-phonon (DPP) etching results in the selective etching of smaller scale structures in comparison with conventional photochemical etching. This information therefore supports the use of the DPP etching method for the surface finishing of organic materials. In future, by combining both DPP and conventional photochemical etching, a further decrease in the $R_{a}$ value could be realized depending on the $R_{a}$ value of the substance in question.

\section{Conflict of Interests}

The authors declare that there is no conflict of interests regarding the publication of this paper.

\section{Acknowledgments}

The authors wish to express special thanks to Dr. Takashi Morimoto and Mr. Yoshinori Tabata (SIGMA Koki Co., Ltd.) for their active support and discussions. This work was partially supported by a Grant-in-Aid for Scientific Research (B) (no. 26286022), the Exploratory Research program (no. 26630122) of MEXT, Core-to-Core Program of JSPS (A. Advanced Research Networks), and a research grant (Basic Research) from The Asahi Glass Foundation. They would like to thank Editage (http://www.editage.jp/) for English language editing.

\section{References}

[1] M. Kaltenbrunner, T. Sekitani, J. Reeder et al., "An ultralightweight design for imperceptible plastic electronics," Nature, vol. 499, no. 7459, pp. 458-463, 2013.

[2] B.-K. Lee, D. S. Kim, and T. H. Kwon, "Replication of microlens arrays by injection molding," Microsystem Technologies, vol. 10, no. 6-7, pp. 531-535, 2004.

[3] L. Jianfeng and D. A. Dornfeld, "Material removal mechanism in chemical mechanical polishing: theory and modeling," IEEE Transactions on Semiconductor Manufacturing, vol. 14, no. 2, pp. 112-133, 2001.

[4] A. J. Kadaksham, B. Lee, M. House, T. Laursen, B. Niekrewicz, and A. Rastegar, "Current status of EUV mask blanks and LTEM substrates defectivity and cleaning of blanks exposed in EUV ADT," in Extreme Ultraviolet (EUV) Lithography II, 79690Z, vol. 7969 of Proceedings of SPIE, San Jose, Calif, USA, February 2011.

[5] T. Yatsui, K. Hirata, W. Nomura, Y. Tabata, and M. Ohtsu, "Realization of an ultra-flat silica surface with angstromscale average roughness using nonadiabatic optical near-field etching," Applied Physics B: Lasers and Optics, vol. 93, no. 1, pp. $55-57,2008$.

[6] M. Ohtsu, "Dressed photon technology", Nanophotonics, vol. 1, pp. 83-97, 2012.

[7] T. Yatsui, W. Nomura, F. Stehlin, O. Soppera, M. Naruse, and M. Ohtsu, "Challenges in realizing ultraflat materials surfaces," Beilstein Journal of Nanotechnology, vol. 4, no. 1, pp. 875-885, 2013.
[8] T. Yatsui, D. Takeuchi, S. Koizumi et al., "Polarization-controlled dressed-photonphonon etching of patterned diamond structures," Physica Status Solidi (A), vol. 211, no. 10, pp. 2339-2342, 2014.

[9] D. Keilin and E. F. Hartree, "Absorption spectrum of oxygen," Nature, vol. 165, no. 4197, pp. 543-544, 1950.

[10] D. W. Allan, "Statistics of atomic frequency standards," Proceedings of the IEEE, vol. 54, no. 2, pp. 221-230, 1966.

[11] R. Srinivasan and B. Braren, "Ultraviolet laser ablation of organic polymers," Chemical Reviews, vol. 89, no. 6, pp. $1303-$ 1316, 1989. 

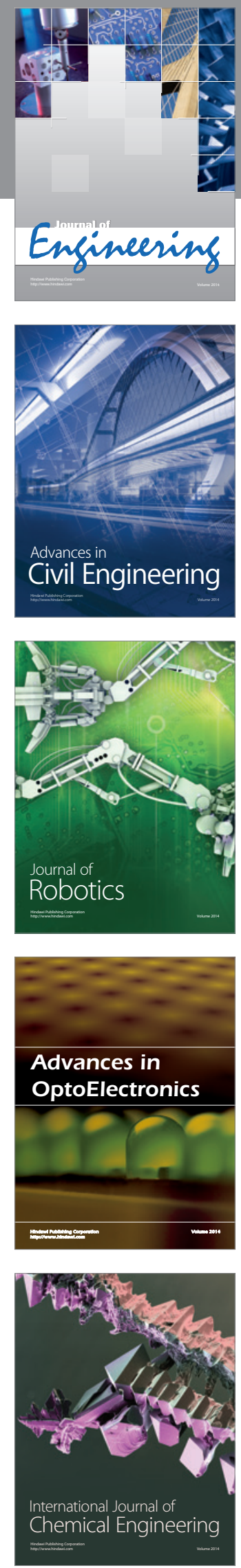

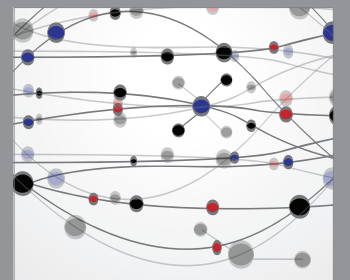

The Scientific World Journal
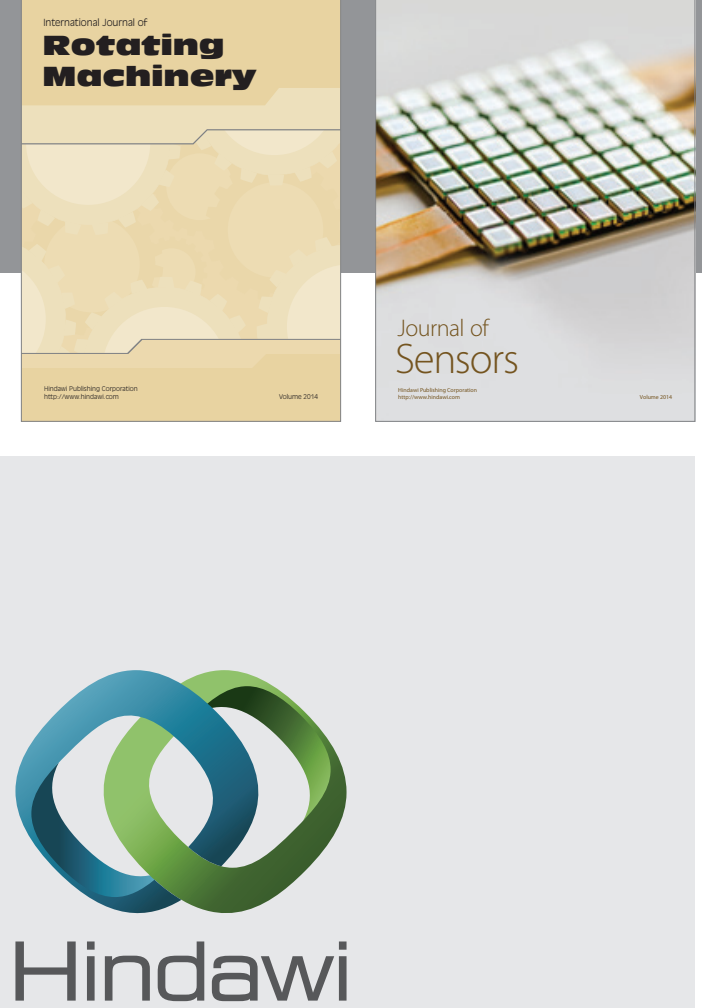

Submit your manuscripts at http://www.hindawi.com
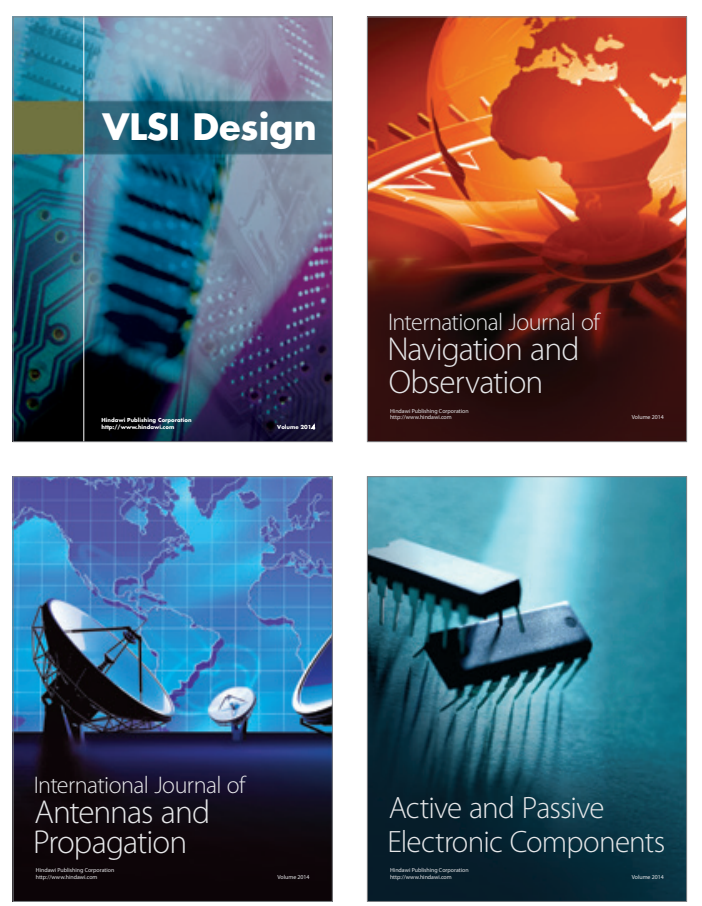
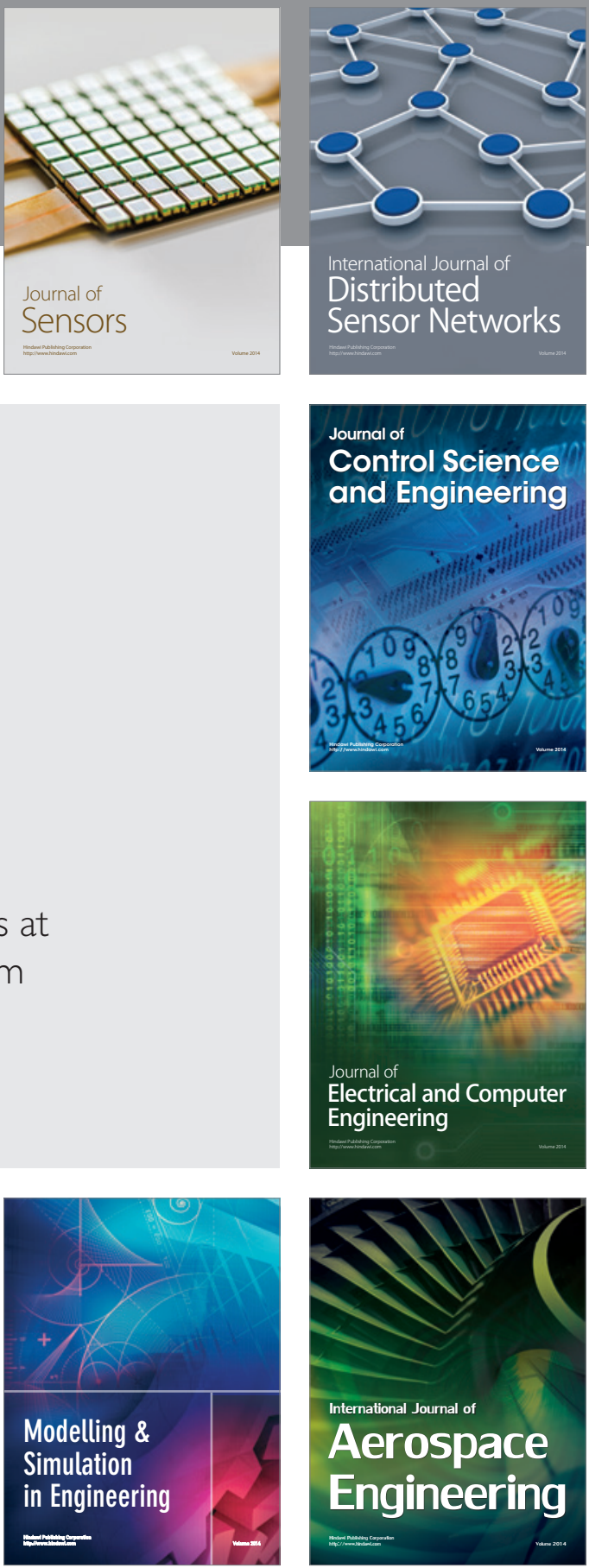

Journal of

Control Science

and Engineering
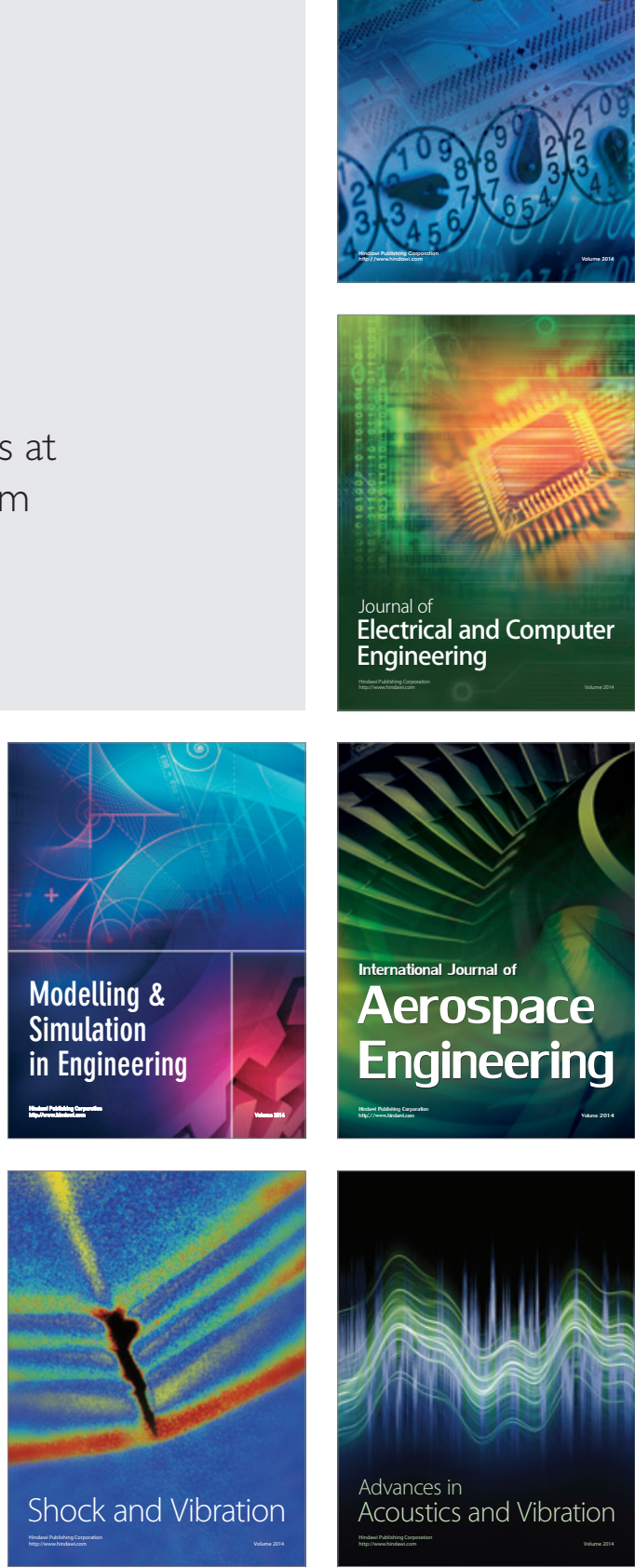